\title{
Dynamical Analysis of a Fractional Order HIV/AIDS Model
}

\author{
Septiangga Van Nyek Perdana Putra ${ }^{1}$, Agus Suryanto ${ }^{2}$, Nur Shofianah ${ }^{3}$ \\ ${ }^{1}$ Departement of Mathematics, Postgraduate Program, Brawijaya University, Indonesia \\ 2,3 Departement of Mathematics, Brawijaya University, Indonesia \\ 13septiangga.vnpp@gmail.com, 2agussuryanto@yahoo.com, ${ }^{3}$ nur shofianah@ub.ac.id
}

\begin{tabular}{|c|c|}
\hline & ABSTRACT \\
\hline Article History: & This article discusses a dynamical analysis of the fractional-order model of \\
\hline $\begin{array}{ll}\text { Received } & : 15-10-2020 \\
\text { Revised } & : 08-11-2020 \\
\text { Accepted } & : 12-11-2020 \\
\text { Online } & : 15-04-2021\end{array}$ & $\begin{array}{l}\text { HIV/AIDS. Biologically, the rate of subpopulation growth also depends on all } \\
\text { previous conditions/memory effects. The dependency of the growth of } \\
\text { subpopulations on the past conditions is considered by applying fractional } \\
\text { derivatives. The model is assumed to consist of susceptible, HIV infected, HIV } \\
\text { infected with treatment, resistance, and AIDS. The fractional-order model of }\end{array}$ \\
\hline Keyword: & $\begin{array}{l}\text { HIV/AIDS with Caputo fractional-order derivative operators is constructed and } \\
\text { then, the dynamical analysis is performed to determine the equilibrium points, }\end{array}$ \\
\hline $\begin{array}{l}\text { Fractional-Order; } \\
\text { PECE Method; } \\
\text { HIV/AIDS; } \\
\text { Epidemic Model; }\end{array}$ & $\begin{array}{l}\text { local stability and global stability of the equilibrium points. The dynamical } \\
\text { analysis results show that the model has two equilibrium points, namely the } \\
\text { disease-free equilibrium point and endemic equilibrium point. The disease-free } \\
\text { equilibrium point always exists and is globally asymptotically stable when the }\end{array}$ \\
\hline 口ist回 & $\begin{array}{l}\text { basic reproduction number is less than one. The endemic equilibrium point exists } \\
\text { if the basic reproduction number is more than one and is globally asymptotically } \\
\text { stable unconditionally. To illustrate the dynamical analysis, we perform some } \\
\text { numerical simulation using the Predictor-Corrector method. Numerical } \\
\text { simulation results support the analytical results. }\end{array}$ \\
\hline
\end{tabular}

dol Crossref
https://doi.org/10.31764/itam.v5i1.3224

\section{A. INTRODUCTION}

Human Immunodeficiency Virus (HIV) is a virus that attacks the human immune system, thus leaving the body vulnerable to various types of diseases. Collection of symptoms of diseases that attack the body that is called the Acquired Immuno Deficiency Syndrome (AIDS). There are several stages of HIV development in the human body. The first stage called the window period is the stage where HIV enters the body and builds antibodies in the body. The second stage is the stage where HIV begins to develop in the body. HIV testing can detect the presence of the virus because antibodies are starting to form. The third stage is the stage where the patient is confirmed to be HIV positive with a declining immune system. The fourth stage or what is called AIDS is the stage where the sufferer is declared positive suffering from AIDS (Kemenkes RI, 2017).

Huo, et al. (2016) constructed a mathematical model of the spread of HIV/AIDS. In this model, the human population is divided into five subpopulations. They assumed that there are subpopulations that change their sexual habits, where this change in behavior aims to reduce the chance of spreading HIV through sexual contact so that the subpopulation will not be 
infected with HIV/AIDS by sexual contact. Using those assumptions, they proposed the following HIV/AIDS model

$$
\begin{aligned}
& \frac{d S}{d t}=\Lambda-\beta S I-\mu S-d S \\
& \frac{d I}{d t}=\beta S I+\alpha_{1} T-d I-k_{1} I-k_{2} I \\
& \frac{d A}{d t}=k_{1} I+\alpha_{2} T-\left(\delta_{1}+d\right) A \\
& \frac{d T}{d t}=k_{2} I-\alpha_{1} T-\left(d+\alpha_{2}+\delta_{2}\right) T \\
& \frac{d R}{d t}=\mu S-d R
\end{aligned}
$$

Table 1. Description of variables and parameters of the system (1)

\begin{tabular}{cl}
$\begin{array}{c}\text { Variables and } \\
\text { parameters }\end{array}$ & \multicolumn{1}{c}{ Description } \\
\hline$S$ & The density of susceptible subpopulation. \\
\hline$I$ & The density of HIV-positive subpopulation in the stage of HIV infection. \\
\hline$A$ & $\begin{array}{l}\text { The density of subpopulation with full-blown AIDS but not receiving ARV } \\
\text { treatment. }\end{array}$ \\
\hline$T$ & The density of subpopulation being treated. \\
\hline$R$ & The density of resistant subpopulation. \\
\hline$\beta$ & New recruitment rate. \\
\hline$\mu$ & The rate of interaction between susceptible and infected by sexual contact. \\
\hline$k_{1}$ & The transfer rate from $S$ to $R$. \\
\hline$k_{2}$ & The transfer rate from $I$ to $A$. \\
\hline$\alpha_{1}$ & The transfer rate from $I$ to $T$. \\
\hline$\alpha_{2}$ & The transfer rate from $T$ to $I$. \\
\hline$\delta_{1}$ & The disease-induced death rate for subpopulations in $A$. \\
\hline$\delta_{2}$ & The disease-induced death rate for subpopulations in $T$. \\
\hline
\end{tabular}

In classical calculus, a function can be derived or integrated once, twice, and so on. Then a question arises regarding the fractional order of derivatives and integrals. Therefore, an idea arises which is the development of classical calculus, namely fractional calculus. The fractional differential equation system was obtained from a nonlinear differential equation system, so that the first derivative of the differential equation system became a fractional-order of the orde- $\alpha$ with $0<\alpha<1$ (Das \& Gupta, 2011).

Biologically, the rate of growth also depends on previous conditions. The fractional differential equation system model is considered to be more suitable in describing phenomena that occur in nature because the subsequent conditions in the model depend not only on the current conditions but also on all the previous conditions. As a result, fractional derivatives at certain points contain information about functions at the previous points.

Several studies have been conducted related to fractional material modeling. For example, related mathematical model of fractional-order conducted by Rihan (2013) . In that study, Rihan developed a mathematical model of the immunity of patients with tumors and HIV and the relationships that affect the growth of these diseases in the body. Other research related to fractional-order mathematical models was conducted by Pinto and Carvalho (2015). Pinto 
and Carvalho form a mathematical model of the effect of drugs on HIV development in the body. Other research related to fractional-order mathematical models can be seen at (Nyabadza et al., 2011), (Rihan et al., 2014), (S.M. \& A.M., 2017), (Hassouna et al., 2018), (Shaikh \& Sooppy Nisar, 2019), (Solís-Pérez et al., 2019), and (Sweilam et al., 2020).

Recently, Silva and Torres (2017) proposed a mathematical model for the spread of HIV/AIDS where the human population is divided into four subpopulations, namely susceptible individuals, HIV-infected individuals with no AIDS symptoms, HIV-infected individuals under ART treatment, and HIV-infected individuals with AIDS symptoms (Silva \& Torres, 2017). Then, they modified their model into a fractional-order to include the memory effects. The dynamical analysis of the obtained fractional-order mathematical model has been conducted and different interpretation has been give by using multiple value parameter fractionals (Silva \& Torres, 2019).

In 2019, Moore, et al. modified the model of Huo, et al. (2016) by modifying the original model into a fractional-order differential equation. They replaced the first-order derivatives by the Caputo-Fabrizio operator of fractional-order derivative operator where its kernel is of the form of the non-singular exponentially decreasing. Moore, et al. (2019) investigated the dynamics of the Caputo-Fabrizio fractional differential equation model for HIV/AIDS with an antiretroviral treatment compartment. They determined the equilibrium points of the model and the conditions for local asymptotic stability of the disease-free equilibrium point. A threestep fractional Adams-Bashforth scheme has been derived and used to obtain numerical solutions of the fractional system.

In this article, we will modify the model developed by Huo, et al. (2016) by replacing the first-order derivatives with the Caputo operator of fractional-order derivative. The fundamental difference between this article and Moore, et al. (2019) lies in the operator of the fractional derivative and the numerical scheme for simulation. In this article we perform a dynamical analysis to investigate the local and global stability of the disease-free equilibrium point and the endemic equilibrium point. Then numerical simulations are performed using the Predictor-Corrector (PECE) method to support the results of the fractional dynamic analysis.

This paper is composed of 4 sections. Section A describes the phases of development of HIV/AIDS, mathematical models epidemiology of HIV/AIDS, and also some research on fractional-order mathematical models related to HIV/AIDS. In section B, we construct the fractional order HIV/AIDS model and determine the point of disease-free equilibrium as well as an endemic equilibrium point. In section $\mathrm{C}$, local and global stability analysis are given and some numerical simulations are performed. Finally, we end up the paper by presenting conclusion in section D.

\section{B. METHODS}

This section describes the model of the spread of HIV/AIDS at fractional-order. The first step is to modify the model of Huo, et al. (2016) to get a fractional-order mathematical model, namely by introducing the Caputo fractional-order derivative operators, and then perform dynamical analysis for the model. 


\section{The Caputo Fractional-Order Formula}

Definition 1 Let $\alpha \in(0,1]$ and $f \in C^{n}([0,+\infty), \mathbb{R})$, and $\Gamma(\cdot)$ is the Gamma function. The Caputo fractional-order derivative defined by

$$
D_{*}^{\alpha} f(t)=\frac{1}{\Gamma(1-\alpha)} \int_{0}^{t}(t-s)^{-\alpha} f^{\prime}(s) d s, t \geq 0 .
$$

(Diethelm, 2010)

By using Definition 1, we obtain the Caputo fractional-order HIV/AIDS model as follows

$$
\begin{aligned}
& D_{*}^{\alpha} S(t)=\Lambda-\beta S I-\mu S-d S, \\
& D_{*}^{\alpha} I(t)=\beta S I+\alpha_{1} T-d I-k_{1} I-k_{2} I, \\
& D_{*}^{\alpha} A(t)=k_{1} I+\alpha_{2} T-\left(\delta_{1}+d\right) A, \\
& D_{*}^{\alpha} T(t)=k_{2} I-\alpha_{1} T-\left(d+\alpha_{2}+\delta_{2}\right) T, \\
& D_{*}^{\alpha} R(t)=\mu S-d R .
\end{aligned}
$$

\section{The Equilibrium Points}

The first step in dynamical analysis is determining all possible equilibrium points of the model. To find the equilibrium point of system (3), the following definition is used.

Definition $2 \bar{x}$ is called an equilibrium point of the system (3) if it satisfies

$$
f(\bar{x})=0, \bar{x} \in \mathbb{R}^{n} \text { for each } t \in \mathbb{R} \text {. }
$$

(Diethelm, 2010)

Based on Definition 2, the equilibrium point of system (3) is attained by solving

$$
\begin{aligned}
& \Lambda-\beta S I-\mu S-d S=0, \\
& \beta S I+\alpha_{1} T-d I-k_{1} I-k_{2} I=0, \\
& k_{1} I+\alpha_{2} T-\left(\delta_{1}+d\right) A=0, \\
& k_{2} I-\alpha_{1} T-\left(d+\alpha_{2}+\delta_{2}\right) T=0, \\
& \mu S-d R=0 .
\end{aligned}
$$

Therefore, we obtain two equilibrium points as follows.

(i) The disease-free equilibrium point

$$
\begin{aligned}
E_{0} & =\left(S^{0}, I^{0}, A^{0}, T^{0}, R^{0}\right) \\
& =\left(\frac{\Lambda}{(\mu+d)}, 0,0,0, \frac{\mu \Lambda}{d(\mu+d)}\right),
\end{aligned}
$$

which always exists.

(ii) The endemic equilibrium point

$E_{1}=\left(S^{*}, I^{*}, A^{*}, T^{*}, R^{*}\right)$,

where

$$
\begin{aligned}
S^{*} & =\frac{\Lambda}{\left(\beta I^{*}+\mu+d\right)}, \\
I^{*} & =\frac{\left(\mathcal{R}_{0}-1\right)(\mu+d)}{\beta}, \\
A^{*} & =\frac{\left(\left(\alpha_{1}+\alpha_{2}+\delta_{2}+d\right) k_{1}+\alpha_{2} k_{2}\right) I^{*}}{\left(\delta_{1}+d\right)\left(\alpha_{1}+\alpha_{2}+\delta_{2}+d\right)}, \\
T^{*} & =\frac{k_{2} I^{*}}{\alpha_{1}+\alpha_{2}+\delta_{2}+d},
\end{aligned}
$$




$$
R^{*}=\frac{\mu \Lambda}{d\left(\beta I^{*}+\mu+d\right)},
$$

with

$$
\mathcal{R}_{0}=\frac{\beta \Lambda\left(\alpha_{1}+\alpha_{2}+\delta_{2}+d\right)}{\left(\left(d+k_{1}+k_{2}\right)\left(\alpha_{1}+\alpha_{2}+\delta_{2}+d\right)-\alpha_{1} k_{2}\right)(\mu+d)} .
$$

The endemic equilibrium point $E_{1}$ exists if $\mathcal{R}_{0}>1$.

\section{RESULT AND DISCUSSION}

This section will discuss the local stability and global stability of each equilibrium point. The local stability is carried out by applying the following theorem.

Theorem 1. The equilibrium points $\bar{x}$ of the system (3) is asymptotically stable if all eigenvalues $\lambda_{j}$ of the Jacobian matrix $J=\frac{\partial \vec{f}}{\partial \vec{x}}$ evaluated at $\bar{x}$ satisfy $\left|\arg \left(\lambda_{n}\right)\right|>\frac{a \pi}{2}$.

(Petras, 2011)

\section{Local Stability}

To perform local stability analysis, the theory of Routh-Hurwitz fractional-order criteria will be used to determine the properties and stability requirements of each equilibrium point.

Theorem 2 The equilibrium points of system (3) with the characteristic equation

$$
P(\lambda)=a_{0} \lambda^{n}+a_{1} \lambda^{n-1}+a_{2} \lambda^{n-2}+\cdots+a_{n}=0,
$$

it is said to be local asymptotic stable if

(i) For $n=1$ then $\left|\arg \left(\lambda_{n}\right)\right|>\frac{a \pi}{2}$ if $a_{1}>0$.

(ii) For $n=2$ then $\left|\arg \left(\lambda_{n}\right)\right|>\frac{a \pi}{2}$ :

$$
\begin{aligned}
& \text { a) If } D(P)>0, a_{1}>0 \text { and } a_{2}>0 \text { or ; } \\
& \text { b)If } D(P)<0, a_{1}<0,4 a_{2}>a_{1}{ }^{2} \text { and }\left|\tan ^{-1}\left(\frac{\sqrt{4 a_{2}-a_{1}^{2}}}{a_{1}}\right)\right|>\frac{\alpha \pi}{2} \text {. }
\end{aligned}
$$

(iii) For $n=3$ then $\left|\arg \left(\lambda_{1,2,3}\right)\right|>\frac{\alpha \pi}{2}$ :
a) If $D(P)>0, a_{1}>0, a_{3}>0, a_{1} a_{2}>a_{3}$, or ;
b)If $D(P)<0, a_{1} \geq 0, a_{2} \geq 0, a_{3}>0$, and $\alpha<\frac{2}{3}$, or ;
c) If $D(P)<0, a_{1}>0, a_{2}>0, a_{1} a_{2}=a_{3}$ for each $0<\alpha<1$,

with

$$
\begin{aligned}
& D(P)=-\left|\begin{array}{ccccc}
1 & a_{1} & a_{2} & a_{3} & 0 \\
0 & 1 & a_{1} & a_{2} & a_{3} \\
3 & 2 a_{1} & a_{2} & 0 & 0 \\
0 & 3 & 2 a_{1} & a_{2} & 0 \\
0 & 0 & 3 & 2 a_{1} & a_{2}
\end{array}\right|, \\
& D(P)=18 a_{1} a_{2} a_{3}+\left(a_{1} a_{2}\right)^{2}-4 a_{3} a_{1}{ }^{2}-4 a_{2}{ }^{2}-27 a_{3}{ }^{2} .
\end{aligned}
$$

(Ahmed et al., 2006) 
a. Local Stability of $E_{0}$

The Jacobian matrix at the equilibrium point $E_{0}$ is given by

$$
J\left(E_{0}\right)=\left(\begin{array}{ccccc}
-(\mu+d) & \frac{-\beta \Lambda}{\mu+d} & 0 & 0 & 0 \\
0 & \frac{\beta \Lambda}{\mu+d}-\left(k_{1}+k_{2}+d\right) & 0 & \alpha_{1} & 0 \\
0 & k_{1} & -\left(\delta_{1}+d\right) & \alpha_{2} & 0 \\
0 & k_{2} & 0 & -\left(\alpha_{1}+\alpha_{2}+\delta_{2}+d\right) & 0 \\
\mu & 0 & 0 & 0 & -d
\end{array}\right) .
$$

The eigenvalues of $J\left(E_{0}\right)$ can be found by solving the characteristic equation $\left|J\left(E_{0}\right)-\lambda I\right|=$ 0 . By cofactor expansion method, it can be shown that the eigenvalues of the matrix satisfy the following equation

$$
(-d-\lambda)\left(-\left(\delta_{1}+d\right)-\lambda\right)(-(\mu+d)-\lambda) \operatorname{det}(A)=0
$$

where

$$
A=\left(\begin{array}{cc}
\frac{\beta \Lambda}{\mu+d}-\left(k_{1}+k_{2}+d\right) & \alpha_{1} \\
k_{2} & -\left(\alpha_{1}+\alpha_{2}+\delta_{2}+d\right)
\end{array}\right)
$$

It is clear that the first three eigenvalues of $J\left(E_{0}\right)$ are $\lambda_{1}=-d<0, \lambda_{2}=-\left(\delta_{1}+d\right)<0$ and $\lambda_{3}=-(\mu+d)<0$, which satisfy $\arg \left(\lambda_{j}\right)=\pi>\frac{a \pi}{2}, i=1,2,3$. Therefore, the stability of the equilibrium point $E_{0}$ is determined by $\lambda_{j}, j=4,5$, which are the eigenvalues of $A$. It is easy to show that the characteristic equation of $A$ is

$$
\lambda^{2}+a_{1} \lambda+a_{2}=0
$$

where

$$
\begin{aligned}
& a_{1}=\left(\left(k_{1}+k_{2}+d\right)+\left(\alpha_{1}+\alpha_{2}+\delta_{2}+d\right)-\frac{\beta \Lambda}{\mu+d}\right) \\
& a_{2}=-\left(\frac{\beta \Lambda}{\mu+d}-\left(k_{1}+k_{2}+d\right)\right)\left(\alpha_{1}+\alpha_{2}+\delta_{2}+d\right)-\alpha_{1} k_{2} .
\end{aligned}
$$

Therefore, the roots are

$$
\lambda_{4,5}=\frac{-a_{1} \pm \sqrt{\mathcal{D}}}{2}
$$

where

$$
\begin{aligned}
\mathcal{D} & =a_{1}^{2}-4 a_{2}, \\
& =\left(\frac{\beta \Lambda}{\mu+d}-\left(k_{1}+k_{2}+d\right)+\left(\alpha_{1}+\alpha_{2}+\delta_{2}+d\right)\right)^{2}+4 \alpha_{1} k_{2}>0 .
\end{aligned}
$$

Since $\mathcal{D}>0$, it is clear that $\lambda_{4,5} \in \mathbb{R}$. The equilibrium point $E_{0}$ is asymptotically stable only when $a_{1}>0$ and $a_{2}>0$. Condition $a_{2}>0$ is equivalent to

$$
\begin{aligned}
& -\left(\frac{\beta \Lambda}{\mu+d}-\left(k_{1}+k_{2}+d\right)\right)\left(\alpha_{1}+\alpha_{2}+\delta_{2}+d\right)-\alpha_{1} k_{2}>0, \\
& \frac{\beta \Lambda}{\mu+d}<\left(k_{1}+k_{2}+d\right)-\frac{\alpha_{1} k_{2}}{\left(\alpha_{1}+\alpha_{2}+\delta_{2}+d\right)}
\end{aligned}
$$




$$
\frac{\beta \Lambda\left(\alpha_{1}+\alpha_{2}+\delta_{2}+d\right)}{\left(\left(k_{1}+k_{2}+d\right)\left(\alpha_{1}+\alpha_{2}+\delta_{2}+d\right)-\alpha_{1} k_{2}\right)(\mu+d)}<1 .
$$

So the equilibrium point $E_{0}$ is local asymptotically stable if $a_{1}>0$ and $\mathcal{R}_{0}<1$.

b. Local Stability of $E_{1}$

When the Jacobian matrix is evaluated at the equilibrium point $E_{1}$, then we get

$$
J\left(E_{1}\right)=\left(\begin{array}{ccccc}
-\beta I^{*}-(\mu+d) & -\beta S^{*} & 0 & 0 & 0 \\
\beta I^{*} & \beta S^{*}-\left(k_{1}+k_{2}+d\right) & 0 & \alpha_{1} & 0 \\
0 & k_{1} & -\left(\delta_{1}+d\right) & \alpha_{2} & 0 \\
0 & k_{2} & 0 & -\left(\alpha_{1}+\alpha_{2}+\delta_{2}+d\right) & 0 \\
\mu & 0 & 0 & 0 & -d
\end{array}\right) .
$$

It can be shown that the eigenvalues of $J\left(E_{1}\right)$ satisfy the characteristic equation

where

$$
(-d-\lambda)\left(-\left(\delta_{1}+d\right)-\lambda\right) \operatorname{det}(B)=0
$$

$$
B=\left(\begin{array}{ccc}
-\beta I^{*}-(\mu+d) & -\beta S^{*} & 0 \\
\beta I^{*} & \beta S^{*}-\left(k_{1}+k_{2}+d\right) & \alpha_{1} \\
0 & k_{2} & -\left(\alpha_{1}+\alpha_{2}+\delta_{2}+d\right)
\end{array}\right)
$$

From the above equation, it is obtained that $\lambda_{1}=-d<0$, and $\lambda_{2}=-\left(\delta_{1}+d\right)<0$. Hence, $\arg \left(\lambda_{j}\right)=\pi>\frac{a \pi}{2}, j=1,2$. Therefore, the stability of the equilibrium point is determined by $\lambda_{j}, j=3,4,5$, which is the eigenvalue of the matrix $B$. The characteristic equation of $B$ is

with

$$
\lambda^{3}+b_{1} \lambda^{2}+b_{2} \lambda+b_{3}=0
$$

$$
\begin{gathered}
b_{1}=\beta I^{*}+(\mu+d)-\beta S^{*}+\left(k_{1}+k_{2}+d\right)+\left(\alpha_{1}+\alpha_{2}+\delta_{2}+d\right), \\
b_{2}=\beta S^{*} \beta I^{*}-\left(\beta I^{*}+(\mu+d)-\beta S^{*}+\left(k_{1}+k_{2}+d\right)\right)\left(\alpha_{1}+\alpha_{2}+\delta_{2}+d\right) \\
+\left(-\beta I^{*}-(\mu+d)\right)\left(\beta S^{*}-\left(k_{1}+k_{2}+d\right)\right)+\alpha_{1} k_{2}, \\
b_{3}=-\beta S^{*} \beta I^{*}\left(\alpha_{1}+\alpha_{2}+\delta_{2}+d\right) \\
-\left(\left(\beta I^{*}+(\mu+d)\right)\left(\beta S^{*}-\left(k_{1}+k_{2}+d\right)\right)\left(\alpha_{1}+\alpha_{2}+\delta_{2}+d\right)\right) \\
+\left(\beta I^{*}+(\mu+d)\right) \alpha_{1} k_{2} .
\end{gathered}
$$

Based on Theorem 2, the roots of the characteristic equation satisfy $\left|\arg \left(\lambda_{n}\right)\right|>\frac{a \pi}{2}$ when :

a) If $D(P)>0, a_{1}>0, a_{3}>0, a_{1} a_{2}>a_{3}$, or ;

b) If $D(P)<0, a_{1} \geq 0, a_{2} \geq 0, a_{3}>0$, and $\alpha<\frac{2}{3}$, or ;

c) If $D(P)<0, a_{1}>0, a_{2}>0, a_{1} a_{2}=a_{3}$ for each $0<\alpha<1$,

with

$$
D(P)=18 b_{1} b_{2} b_{3}+\left(b_{1} b_{2}\right)^{2}-4 b_{3}{b_{1}}^{2}-4{b_{2}}^{2}-27{b_{3}}^{2} \text {. }
$$

It can be seen that $b_{3}>0$ is a necessary condition for the equilibrium point $E_{1}$ to be stable. 
Conditions $b_{3}>0$ is equivalent to

$$
\begin{aligned}
\beta S^{*} \beta I^{*}\left(\alpha_{1}+\alpha_{2}+\delta_{2}+d\right)-\left(\left(\beta I^{*}+(\mu+d)\right)\left(\beta S^{*}-\left(k_{1}+k_{2}+d\right)\right)\left(\alpha_{1}+\alpha_{2}+\delta_{2}+d\right)\right) \\
-\left(\beta I^{*}+(\mu+d)\right) \alpha_{1} k_{2}>0 .
\end{aligned}
$$

By substituting values of $I^{*}$ and $S^{*}$ into the above equation, we get

$$
\mathcal{R}_{0}\left(\left(k_{1}+k_{2}+d\right)\left(\alpha_{1}+\alpha_{2}+\delta_{2}+d\right)-\alpha_{1} k_{2}\right)\left(1-\frac{1}{\mathcal{R}_{0}}\right)>0 .
$$

Thus, $b_{3}>0$ is achieved only if $\mathcal{R}_{0}>1$.

\section{Global Stability}

To perform a global stability analysis of a fractional-order systems, the following theorems and lemmas are needed

Theorem 3. Suppose that $\Omega$ is a closed and finite set, each solution of system (3) with initial values $x\left(t_{0}\right) \in \Omega$ and $\forall x(t) \in \Omega$. If $\exists V(x): \mathbb{R}^{n} \rightarrow \mathbb{R}$ with the first partial derivative is continuous, it satisfies the following conditions :

$$
D^{\alpha} V(x) \leq 0 \text {. }
$$

(Vargas-De-León, 2015)

Lemma 1. Suppose $x(t) \in C([0,+\infty))$. If $x(t)$ satisfies $D_{*}^{\alpha} x \leq \Lambda-(\mu+d) x(t), x(0)=x_{0} \in$ $\mathbb{R}, 0<\alpha<1$, then $x(t) \leq\left(x_{0}-\frac{\Lambda}{(\mu+d)}\right) E_{\alpha}\left[-(\mu+d) t^{\alpha}\right]+\frac{\Lambda}{(\mu+d)}$ and

$$
\lim _{t \rightarrow \infty} S(t) \leq \frac{\Lambda}{(\mu+d)} .
$$

(Li et al., 2017)

Theorem 4. Suppose that $x(t) \in \mathbb{R}$ is a continuous function and its Caputo fractional derivative exists for $0<\alpha<1$. For any time $t \geq t_{0}, x(t)$ satisfy

$$
D_{*}^{\alpha}\left[x(t)-x^{*}-x^{*} \ln \frac{x(t)}{x^{*}}\right] \leq\left(1-\frac{x^{*}}{x(t)}\right) D_{*}^{\alpha} x(t), \quad x^{*} \in \mathbb{R}, \forall \alpha \in(0,1) .
$$

(Vargas-De-León, 2015)

a. Global Stability of $E_{0}$

Consider a Lyapunov function, which is defined as

$$
V(t)=I+m T \text {, }
$$

where

$$
m=\frac{\alpha_{1}}{\left(\alpha_{1}+d+\delta_{2}+\alpha_{2}\right)}
$$

The fractional derivative of $(5)$ is

$$
D^{\alpha} V(t)=\left(\beta S-\left(d+k_{1}+k_{2}\right)+m k_{2}\right) I+\left(\alpha_{1}-m\left(\alpha_{1}+d+\alpha_{2}+\delta_{2}\right)\right) T .
$$

Based on Lemma 1, (6) can be written as follows

$$
D^{\alpha} V(t)=\left(\beta S-\left(d+k_{1}+k_{2}\right)+m k_{2}\right) I+\left(\alpha_{1}-m\left(\alpha_{1}+d+\alpha_{2}+\delta_{2}\right)\right) T,
$$




$$
\leq\left(\frac{\beta \Lambda}{\mu+d}-\left(d+k_{1}+k_{2}\right)+m k_{2}\right) I+\left(\alpha_{1}-m\left(\alpha_{1}+d+\alpha_{2}+\delta_{2}\right)\right) T .
$$

Then by substituting the value of $m$ into the equation above, we obtain

$$
D^{\alpha} V(t) \leq\left(\frac{\left(\mathcal{R}_{0}-1\right)\left(\left(d+k_{1}+k_{2}\right)\left(\alpha_{1}+d+\alpha_{2}+\delta_{2}\right)-\alpha_{1} k_{2}\right)}{\left(\alpha_{1}+d+\alpha_{2}+\delta_{2}\right)}\right) I .
$$

Since all parameters are positive then that $D^{\alpha} V(t)<0$ if $\mathcal{R}_{0}<1$. If $\mathcal{R}_{0}=1$ and/or $I=$ 0 such that $D^{\alpha} V(t)=0$. Hence, the equilibrium point $E_{0}$ is globally asymptotically stable if $\mathcal{R}_{0}<1$.

b. Global Stability of $E_{1}$

We first define a Lyapunov function as follows

$$
V(t)=S-S^{*}-S^{*} \ln \frac{S}{S^{*}}+\left(I-I^{*}-I^{*} \ln \frac{I}{I^{*}}\right)+\frac{\alpha_{1} T^{*}}{k_{2} I^{*}}\left(T-T^{*}-T^{*} \ln \frac{T}{T^{*}}\right) .
$$

Based on Theorem 4, it is obtained

$$
D^{\alpha} V(t) \leq\left(1-\frac{S^{*}}{S}\right) D^{\alpha} S+\left(1-\frac{I^{*}}{I}\right) D^{\alpha} I+\frac{\alpha_{1} T^{*}}{k_{2} I^{*}}\left(1-\frac{T^{*}}{T}\right) D^{\alpha} T .
$$

Let $x=\frac{S}{S^{*}}, \quad y=\frac{I}{I^{*}}, \quad \mu=\frac{T}{T^{*}}$, then (8) can be written as

$$
D^{\alpha} V(t) \leq-\left(\mu_{1} S^{*}+d S^{*}\right) \frac{(1-x)^{2}}{x}+\beta I^{*} S^{*}\left(2-x-\frac{1}{x}\right)+\alpha_{1} T^{*}\left(2-\frac{\mu}{y}-\frac{y}{\mu}\right) \text {. }
$$

By noticing that all parameters are positive and applying the inequality of arithmetic mean and geometric mean, it is proven $D^{\alpha} V(t) \leq 0$. Furthermore, it can be seen $D^{\alpha} V(t)=0$ if and only if $S=S^{*}, I=I^{*}$, and $T=T^{*}$. Thus, it is concluded that the equilibrium point $E_{1}$ is globally asymptotically stable whenever $E_{1}$ exists.

From the discussion above it can be seen that the disease-free equilibrium point $E_{0}$ is globally stable if $\mathcal{R}_{0}<1$. On the other hand, if the endemic equilibrium point $E_{1}$ exists, namely when $\mathcal{R}_{0}>1$, then $E_{1}$ is globally asymptotically stable. Therefore parameters $\mathcal{R}_{0}$ can be considered as the basic reproduction number.

\section{Numerical Simulations}

In this part we present some numerical simulation using the Predictor-Corrector scheme (Diethelm, 2010). The parameter values used for numerical simulations are based on (Huo et al., 2016) and can be seen in the following Table 2.

Table 2. Parameter values

\begin{tabular}{cc}
\hline Parameter & Parameter values \\
\hline$\Lambda$ & 0.55 \\
\hline$\beta$ & 0.03 \\
\hline$d$ & 0.0196 \\
\hline$k_{2}$ & 0.35 \\
\hline$k_{1}$ & 0.15 \\
\hline$\delta_{1}$ & 0.0909 \\
\hline$\delta_{2}$ & 0.0667 \\
\hline$\mu$ & 0.03 \\
\hline
\end{tabular}


The initial value used in this simulation is $S(0)=35, I(0)=24, T(0)=8, A(0)=15$, and $R(0)=0$.

a. Numerical Simulation I

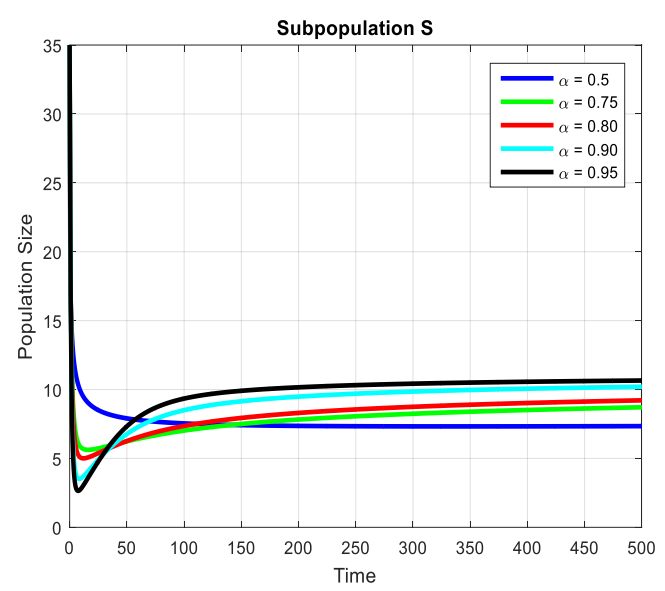

(a)

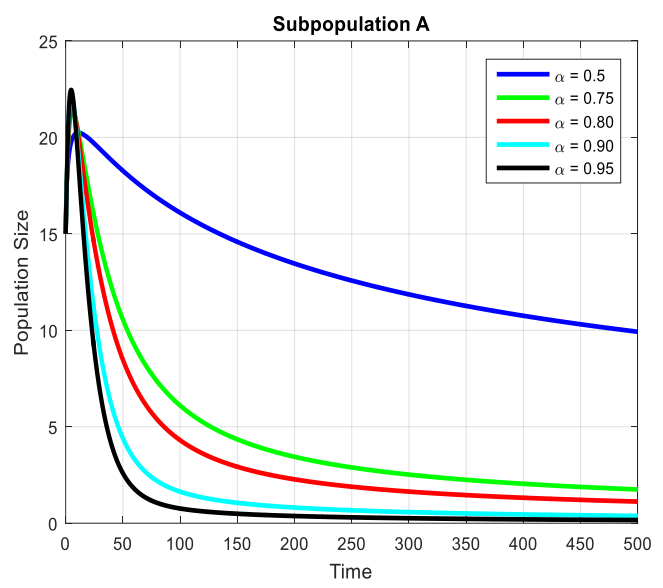

(c)

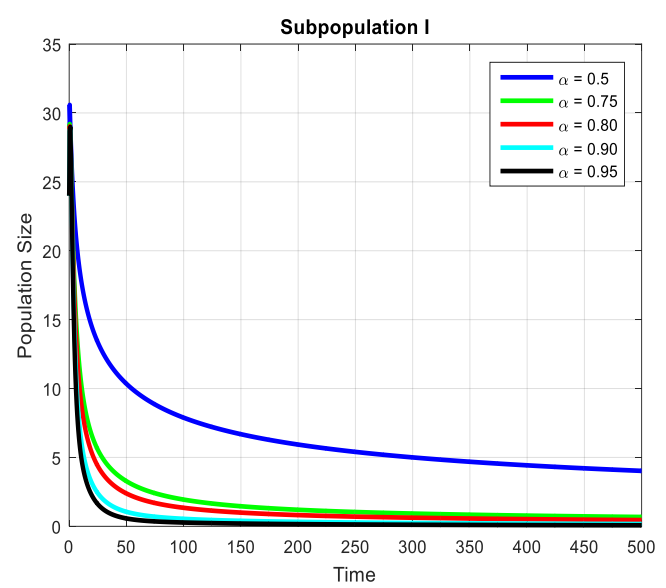

(b)

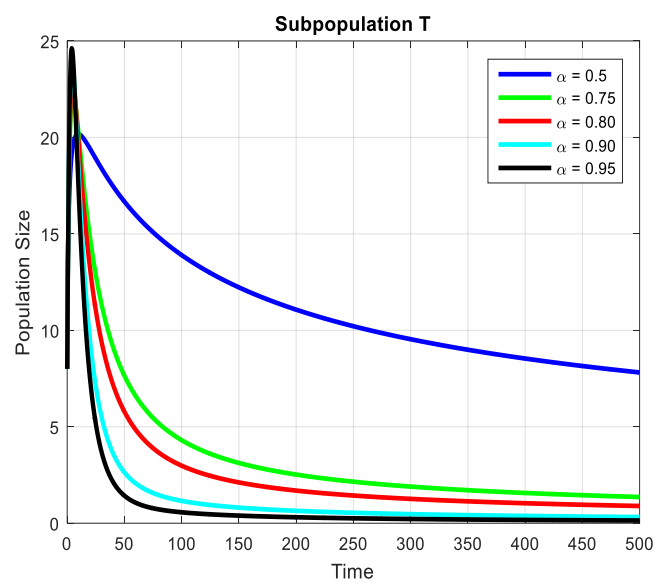

(d)

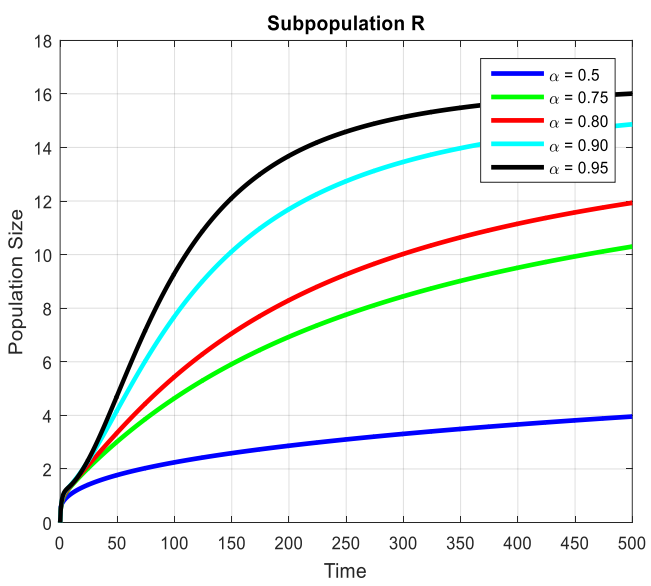

(e)

Figure 1. The behavior of system in numerical simulation I (a) Subpopulation $S$, (b) Subpopulation $I$, (c) Subpopulation $A$, (d) Subpopulation $T$, and (e) Subpopulation $R$. 
Based on the parameter values in Table 2 and $\alpha_{1}=0.08$ and $\alpha_{2}=0.03$, we get $\mathcal{R}_{0}=$ $0.88<1$ and the disease-free equilibrium point $E_{0}=(11.89,0,0,0,16.97)$. It is also found that $a_{1}=0.383>0, a_{2}=0.009>0$, and $\mathcal{D}(P)=0.112>0$. Hence, Theorem 2.(a).(ii) says that the disease-free equilibrium point $E_{0}$ is asymptotically stable for $0<\alpha<1$. Figure 1 illustrates the behavior of the solution of each subpopulation for $0 \leq t \leq 500$ and $\alpha=0.5, \alpha=0.75, \alpha=0.8, \alpha=0.9$, and $\alpha=0.95$. It is seen that using different values of the order of the fractional derivative $(\alpha)$, all numerical solutions are convergent to the disease free equilibrium point $E_{0}$. However, detail observation shows that a solution with larger value of $\alpha$ has faster convergence in approaching the equilibrium point $E_{0}$.

\section{b. Numerical Simulation II}

Next, we perform simulation using parameter values as in Table 1 and $\alpha_{1}=0,25$ and $\alpha_{2}=0,01$. In this case, we get $\mathcal{R}_{0}=1.25>0$ and therefore there exists an endemic equilibrium point, namely $E_{1}=(8.9,0.41,0.41,0.59,13.62)$. The characteristic equation of the Jacobian matrix at $E_{1}$ is given by

$$
\lambda^{3}+b_{1} \lambda^{2}+b_{2} \lambda+b_{3}=0
$$

where $b_{1}=0.661>0, b_{2}=0.040>0, b_{3}=0.001>0$, and $\mathcal{D}(P)=0.005>0$, which shows that $E_{1}$ is asymptotically stable. To see the effect of the order of the fractional derivative $(\alpha)$, we perform numerical simulations using five different values of $\alpha$, namely $\alpha=0.5, \alpha=0.75$, $\alpha=0.8, \alpha=0.9$, and $\alpha=0.95$. The numerical results depicted in Figure 2 show that all numerical solutions with different values of $\alpha$ are convergent to $E_{1}$, confirming that the endemic equilibrium point $E_{1}$ is asymptotically stable. Similar to the previous simulation, it is shown that if the order of the fractional derivative is larger then the solution has faster convergence in approaching the endemic equilibrium point.

Based on the results of numerical simulation I and numerical simulation II, it can be seen that the greater the value of the fractional-order parameter, the faster the numerical solution converges to the equilibrium point. Such behaviour is similar to the result obtained by Moore, et al. (2019).

In this paper we have introduced a fractional order model of the spread of HIV / AIDS. Since the fractional order is a nonlocal operator, the growth rate of each sub-population is dependent on all previous state. In other words, we have include the memory effects in the model. This is in contrast with the original model where the growth rate is modelled by the first order derivative, which means that the growth rate only depends on the current condition. Hence, our model is closer to problems in the real world when compared to mathematical models of integer-order. 


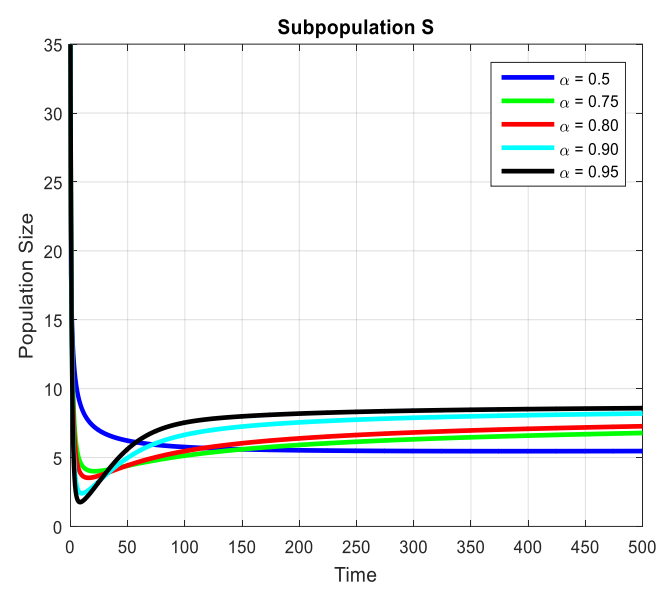

(a)

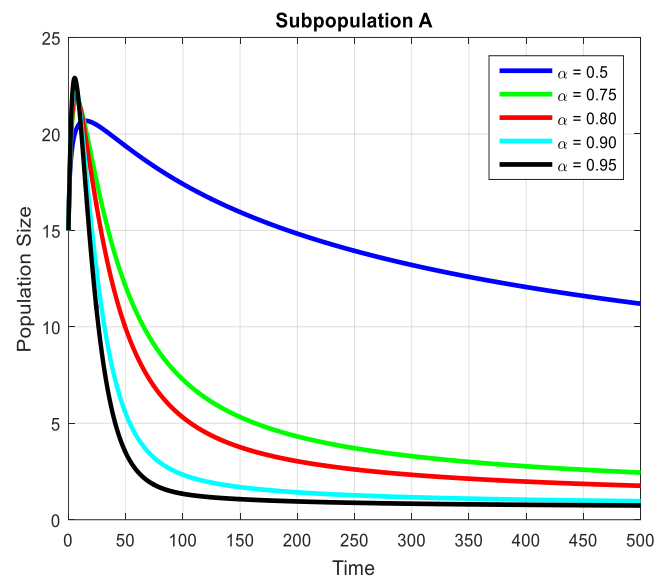

(c)

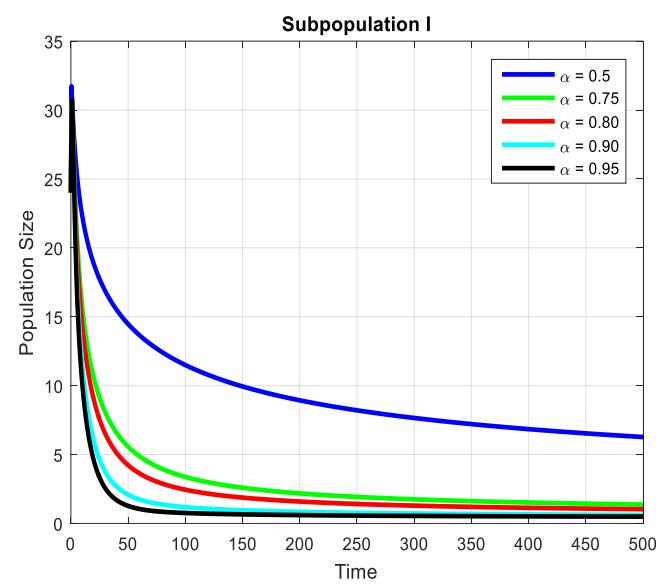

(b)

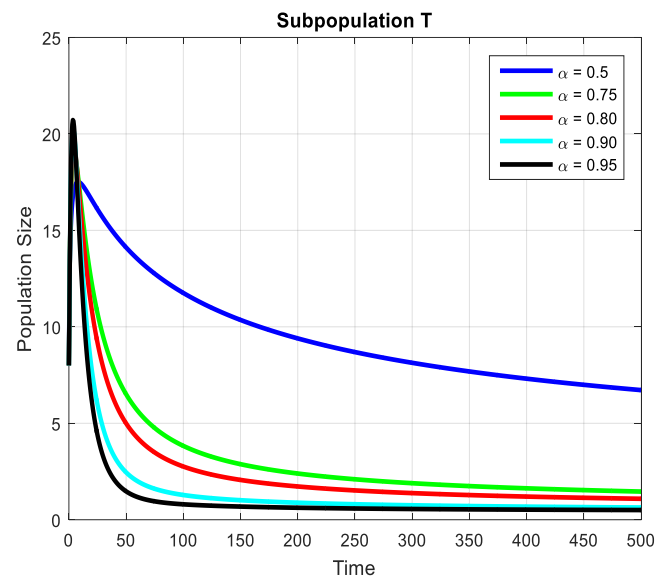

(d)

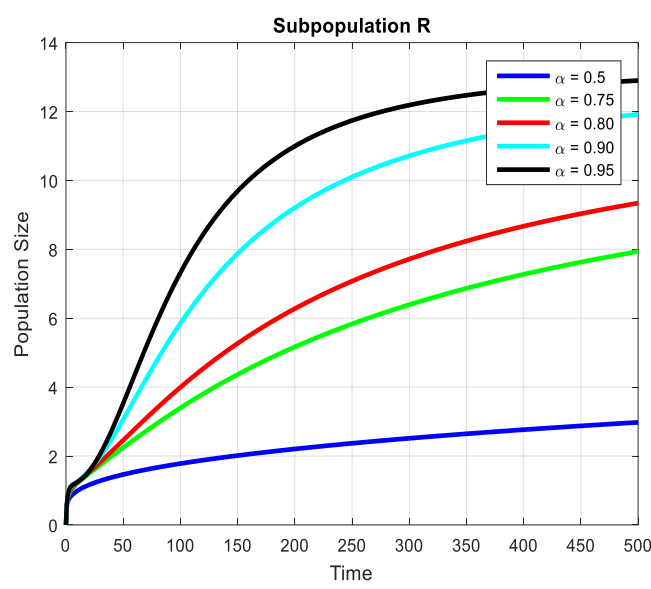

(e)

Figure 2. The behavior of system in numerical simulation I (a) Subpopulation $S$, (b) Subpopulation $I$, (c) Subpopulation $A$, (d) Subpopulation $T$, and (e) Subpopulation $R$.

\section{CONCLUSION AND SUGGESTIONS}

Based on our analytical results, it is concluded that the constructed model has two equilibrium points, namely the disease-free equilibrium point $\left(E_{0}\right)$ and endemic equilibrium point $\left(E_{1}\right)$. The equilibrium point $\left(E_{0}\right)$ always exists and is globally asymptotically stable if the basic reproduction number is less than one $\left(\mathcal{R}_{0}<1\right)$. When $\mathcal{R}_{0}>1$, the equilibrium point 
$\left(E_{1}\right)$ exists and is globally asymptotically stable. The numerical simulation results support the results of dynamical analysis. Based on the results of numerical simulations, it is known that the greater the fractional derivative order, the faster the converges to the equilibrium points.

This article uses the Caputo fractional-order derivative operator to form a fractional-order mathematical model of the spread of HIV / AIDS. In subsequent research, it is recommended to apply the proposed model for the real data.

\section{ACKNOWLEDGEMENT}

The author (Septiangga Van Nyek) appreciates the Assalaam Foundation for providing scholarships to the author.

\section{REFERENCES}

Ahmed, E., El-Sayed, A. M. A., \& El-Saka, H. A. A. (2006). On some Routh-Hurwitz conditions for fractional order differential equations and their applications in Lorenz, Rössler, Chua and Chen systems. Physics Letters, Section A: General, Atomic and Solid State Physics, 358(1), 1-4. https://doi.org/10.1016/j.physleta.2006.04.087

Das, S., \& Gupta, P. K. (2011). A mathematical model on fractional Lotka-Volterra equations. Journal of Theoretical Biology, 277(1), 1-6. https://doi.org/10.1016/j.jtbi.2011.01.034

Diethelm, K. (2010). The Analysis of Fractional Differential Equations. Lecture Notes in Mathematics, 2004, 1-30.

Hassouna, M., Ouhadan, A., \& El Kinani, E. H. (2018). On the solution of fractional order SIS epidemic model. Chaos, Solitons and Fractals, 117, 168-174. https://doi.org/10.1016/j.chaos.2018.10.023

Huo, H. F., Chen, R., \& Wang, X. Y. (2016). Modelling and stability of HIV/AIDS epidemic model with treatment. Applied Mathematical Modelling, 40(13-14), 6550-6559. https://doi.org/10.1016/j.apm.2016.01.054

Kemenkes RI. (2017). Stop HIV AIDS. Kementerian Kesehatan Republik Indonesia, 1-3. http://www.kemkes.go.id/development/site/depkes/pdf.php?id=1-17042500008

Li, H. L., Zhang, L., Hu, C., Jiang, Y. L., \& Teng, Z. (2017). Dynamical analysis of a fractional-order predator-prey model incorporating a prey refuge. Journal of Applied Mathematics and Computing, 54(1-2), 435-449. https://doi.org/10.1007/s12190-016-1017-8

Moore, E. J., Sirisubtawee, S., \& Koonprasert, S. (2019). A Caputo-Fabrizio fractional differential equation model for HIV/AIDS with treatment compartment. Advances in Difference Equations, 2019(1). https://doi.org/10.1186/s13662-019-2138-9

Nyabadza, F., Mukandavire, Z., \& Hove-Musekwa, S. D. (2011). Modelling the HIV/AIDS epidemic trends in South Africa: Insights from a simple mathematical model. Nonlinear Analysis: Real World Applications, 12(4), 2091-2104. https://doi.org/10.1016/j.nonrwa.2010.12.024

Petras, I. (2011). Fractional-Orde Nonlinear System. Springer-Verlag.

Pinto, C. M. A., \& Carvalho, A. R. M. (2015). Effect of drug-resistance in a fractional complex-order model for HIV infection. IFAC-PapersOnLine, 28(1), 188-189. https://doi.org/10.1016/j.ifacol.2015.05.162

Rihan, F. A. (2013). Numerical modeling of fractional-order biological systems. Abstract and Applied Analysis, 2013. https://doi.org/10.1155/2013/816803

Rihan, F. A., Baleanu, D., Lakshmanan, S., \& Rakkiyappan, R. (2014). On fractional SIRC model with salmonella bacterial infection. Abstract and Applied Analysis, 2014. https://doi.org/10.1155/2014/136263

S.M., S., \& A.M., Y. (2017). On a fractional-order model for HBV infection with cure of infected cells. Journal of the Egyptian Mathematical Society, 25(4), 445-451. https://doi.org/10.1016/j.joems.2017.06.003

Shaikh, A. S., \& Sooppy Nisar, K. (2019). Transmission dynamics of fractional order Typhoid fever model using Caputo-Fabrizio operator. Chaos, Solitons and Fractals, 128, 355-365. https://doi.org/10.1016/j.chaos.2019.08.012

Silva, C. J., \& Torres, D. F. M. (2017). Global Stability For a HIV/AIDS Model. Communications Faculty of Sciences Universitiy of Ankara Series A1 Mathematics and Statistics, 1-8. 
Silva, C. J., \& Torres, D. F. M. (2019). Stability of a fractional HIV/AIDS model. Mathematics and Computers in Simulation, 164, 180-190. https://doi.org/10.1016/j.matcom.2019.03.016

Solís-Pérez, J. E., Gómez-Aguilar, J. F., \& Atangana, A. (2019). A fractional mathematical model of breast cancer competition model. Chaos, Solitons and Fractals, 127, 38-54. https://doi.org/10.1016/j.chaos.2019.06.027

Sweilam, N. H., AL-Mekhlafi, S. M., Mohammed, Z. N., \& Baleanu, D. (2020). Optimal control for variable order fractional HIV/AIDS and malaria mathematical models with multi-time delay. Alexandria Engineering Journal, 59(5), 3149-3162. https://doi.org/10.1016/j.aej.2020.07.021

Vargas-De-León, C. (2015). Volterra-type Lyapunov functions for fractional-order epidemic systems. Communications in Nonlinear Science and Numerical Simulation, 24(1-3), 75-85. https://doi.org/10.1016/j.cnsns.2014.12.013 